\title{
Inteligencia Emocional, Felicidad y Enojo, en Relación a la Violencia Psicológica en el Noviazgo de Jóvenes Universitarios
}

\author{
Norma Angélica Ortega-Andrade, \\ Universidad Autónoma del Estado de Hidalgo (UAEH), Mexico \\ María Teresa Dávalos-Romo, \\ Universidad Autónoma de Zacatecas (UAZ), Mexico \\ Ana María Rivera-Guerrero, \\ Universidad Autónoma del Estado de Hidalgo (UAEH), Mexico \\ José María Guajardo-Espinoza, \\ Universidad Autónoma del Estado de Coahuila (UADEC), Mexico
}

Doi:10.19044/esj.2019.v15n28p166 URL:http://dx.doi.org/10.19044/esj.2019.v15n28p166

\section{Resumen}

Estudios relacionados con la violencia psicológica en el noviazgo, refieren que el manejo inadecuado de las emociones puede ser un detonante para violentar o ser violentado, por lo cual, el presente estudio tuvo como objetivo identificar cómo la inteligencia emocional, el nivel de felicidad y el enojo se relacionan con la presencia de violencia en la relación de noviazgo de estudiantes de la Licenciatura en Psicología de dos universidades públicas de México. En total participaron 242 universitarios (139 de una universidad de Hidalgo y 103 de una universidad de Zacatecas). Se empleó la Escala de Inteligencia Emocional (TMMS-24), el Cuestionario de Felicidad de Oxford (OHQ), la Escala de Enojo Rasgo y la Escala de Violencia Psicológica en el Noviazgo. Los resultados evidenciaron que los estudiantes de ambas universidades prestan una adecuada atención a sus emociones como una adecuada regulación emocional, sin embargo, los estudiantes de la universidad de Hidalgo necesitan mejorar su comprensión emocional; así mismo los alumnos de la universidad de Zacatecas son más felices que los alumnos de la universidad de Hidalgo; y en ambos casos presentan bajos niveles de enojo. Los porcentajes de violencia son bajos. La felicidad está vinculada en forma directa con la regulación de las emociones y el enojo se encontró inversamente relacionado con los componentes de la inteligencia emocional, la violencia y la felicidad. 
Palabras claves: Inteligencia emocional, Felicidad, Enojo, Violencia en el noviazgo

\title{
Emotional Intelligence, Happiness and Anger, in Relation to Psychological Violence in the Courtship in College Students
}

\author{
Norma Angélica Ortega-Andrade, \\ Universidad Autónoma del Estado de Hidalgo (UAEH), Mexico \\ María Teresa Dávalos-Romo, \\ Universidad Autónoma de Zacatecas (UAZ), Mexico \\ Ana María Rivera-Guerrero, \\ Universidad Autónoma del Estado de Hidalgo (UAEH), Mexico \\ José María Guajardo-Espinoza, \\ Universidad Autónoma del Estado de Coahuila (UADEC), Mexico
}

\begin{abstract}
Studies related to psychological violence during courtship indicate that improper handling of emotions may lead to be violent or to allow violence. The present study is aimed to identify how emotional intelligence, the level of happiness and anger are related to the presence of violence in the relationship of students of the Bachelor of Psychology of two public universities in Mexico. In total 242 university students participated (139 from a university in Hidalgo and 103 from a university in Zacatecas). The Emotional Intelligence Scale (TMMS-24), the Oxford Happiness Questionnaire (OHQ), the Trait Anger Scale and the Psychological Violence Scale in Dating were used. The results showed that the students of both universities pay adequate attention to their emotions because they have an adequate emotional regulation. However, the students of the University of Hidalgo need to improve their emotional understanding; likewise the students of the University or Zacatecas are happiness that the students of the University of Hidalgo; and in both cases they have low levels of anger. The percentages of violence are low. Happiness is directly linked to the regulation of emotions and anger was inversely related to the components of emotional intelligence, violence and happiness.
\end{abstract}

Keywords: Emotional intelligence, Happiness, Anger, Violence in courtship 


\section{Introducción}

En un mundo donde las condiciones sociales, interpersonales y personales parecen cada vez más estar permeadas de situaciones violentas, demanda la imperiosa necesidad de hallar los posibles factores asociados que proporcionen información de interés para su factible atención. Uno de los hallazgos sobresalientes que pueden contribuir a comprender dicho fenómeno, son los encontrados en los estudios sobre las emociones y la inteligencia emocional asociados al bienestar psicológico como un medio para abordar y dar una respuesta favorable a la violencia en los diferentes contextos y relaciones interpersonales, como puede ser el caso particular del noviazgo en estudiantes universitarios.

La relevancia de las emociones, es que éstas permiten a las personas adaptarse a las condiciones de su entorno, ajustarse y relacionarse acorde a las circunstancias que se estén viviendo; de modo que esta posibilidad brinda a los sujetos un alto grado de control sobre las mismas y cuando esto se alcanza, entonces se dice que se está utilizando la inteligencia emocional (Guerri, 2016).

La Inteligencia Emocional (IE), por lo tanto, es un constructo que hace referencia a la habilidad de las personas para percibir (en él mismo y en los demás) y expresar emociones de forma apropiada; así como la capacidad de usar dicha información para facilitar el pensamiento y regular el comportamiento. Su importancia radica en que ésta puede ayudar a niños, adolescentes y jóvenes a guiar sus pensamientos y autorreflexión, contribuyendo al desarrollo de un estado de bienestar subjetivo (FernándezBerrocal \& Extremera, 2009).

Un estudio llevado a cabo por Blázquez-Alonso, Moreno-Manso, García-Baamonde y Guerrero-Barona (2012) sobre la relación entre la inteligencia emocional y las relaciones interpersonales, reportó una correlación positiva entre la inteligencia emocional, la empatía, el autocontrol, las respuestas cooperativas y el bienestar subjetivo en situaciones sociales y relaciones afectivas como la pareja. De igual forma, reportaron que las relaciones que establecen los jóvenes cuyos recursos emocionales son menores, utilizan en mayor medida la violencia psicológica con su pareja (críticas, insultos, descalificaciones, minusvalorización, hostilidad, desapego, coartación, exigencias desmedidas, ridiculización, culpabilización y manipulación).

\section{La violencia en el noviazgo}

El noviazgo es una condición que involucra la perspectiva de dos personas que coinciden en intereses, necesidades y gustos; se caracteriza por ser una etapa de demostración de emociones y afectos, antes de pasar a un compromiso más específico de vida en pareja o el matrimonio (Pérez, García, 
\& Pérez, 2018). Primordialmente está matizada por una fase de enamoramiento, donde se idealiza a la persona y se busca estar la mayor parte del tiempo con ella, pero cuando esta fase termina, pueden llegan a sufrir desavenencias y dudas, que en ocasiones llegan a convertirse en actos de dominio, control y manipulación hacia uno de los integrantes de la pareja (Izquierdo, 2007).

La violencia en la pareja, por lo tanto, no sólo se extiende a las personas casadas o en convivencia, sino también en los noviazgos o relaciones en donde existe una relación afectiva o sexual. Rey (2008) la define como cualquier comportamiento dentro de una relación íntima que causa daño físico, sexual y psíquico. Cabe destacar que, la violencia psicológica antecede y prevalece ante cualquier tipo de violencia, y que lamentablemente ha sido la menos estudiada y poco clarificada; esto debido a que puede comenzar a manifestarse en etapas iniciales de una relación afectiva de forma sutil, por ello, puede pasar más desapercibida, pero sus objetivos en una relación de pareja son los mismos: pretender el dominio del otro a través de la desvalorización y la implantación de hábitos de sumisión (Alberdi, 2005).

La violencia en el noviazgo contempla cualquier acto, palabra $u$ omisión, que produce daño o lesión, por el cual, una persona trata de doblegar o paralizar a su pareja con el objetivo de dominar y someter ejerciendo el poder, haciendo uso tanto de la fuerza física como psicológica y emocional (Betancourt, López, \& González, 2011; Pozueco, Moreno, Blázquez, \& García-Baamonde, 2013; Rubio-Garay, Carrasco, Amor, \& López-González, 2015). Como parte de las consecuencias que se han detectado en las víctimas, se encuentra la depresión, abuso de sustancias adictivas, intentos de suicidio, depresión, ansiedad y estrés postraumático (Pick \& Givaudan, 2010).

Los mitos o creencias en torno a la relación de pareja, puede ser una de las causas por las cuales los jóvenes llegan a estar en dicha condición. Ideas como "el amor lo puede todo" o creencias fuertemente arraigadas al amor romántico, lleva en muchas ocasiones a que los adolescentes y jóvenes vean las agresiones como algo inherente a la relación, llegando incluso a minimizar o negarlas debido a que en su generalidad son conductas eventuales (RubioGaray, Carrasco, Amor, \& López-González, 2015), por lo que es común que no se dé la ruptura, sino que la relación de noviazgo permanezca.

La violencia en el noviazgo es un fenómeno que se ha incrementado en la población adolescente y joven en los últimos años en México (ValenciaValera \& Vega-López, 2015). Datos reportados por la Encuesta Nacional sobre la Dinámica de las Relaciones en los Hogares 2016, realizada por el Instituto Nacional de Estadística y Geografía, reportan que de los 46.5 millones de mujeres mexicanas de 15 años y más, el $66.1 \%$ han enfrentado al menos un incidente de violencia por parte del agresor alguna vez en su vida. 
El $43 \%$ de las mujeres encuestadas refiere haber sufrido violencia por parte de su actual o última pareja, esposo o novio, durante el tiempo de su relación.

La Organización Mundial de la Salud, ha dado a conocer que 3 de cada 10 adolescentes denuncian que han sufrido violencia en el noviazgo (Mesta, 2016). Esta situación hace hincapié en dos factores de riesgos altamente alarmantes. Primero, que según manifiesta Cornelius y Resseguie (2007) la violencia suele prevalecer e incrementarse hasta un $88 \%$ a lo largo de los años de convivencia, manteniéndose así en la espiral de violencia a lo largo del matrimonio (Batiza, 2017). Y segundo, que, debido a factores familiares, creencias de roles de género, cultura, etc. los jóvenes consideran que dichas conductas son algo natural o normal, sin percatarse de forma clara que están viviendo una relación violenta (Mesta, 2016; Ramírez \& Nuñez, 2010).

Un estudio realizado por Olvera, Arias y Amador en 2010 con estudiantes de la Universidad Autónoma del Estado de México, cuyo objetivo fue identificar si existía violencia en las relaciones de noviazgo, encontró que de 100 participantes, el 92\% de ellos, reconoció indicios de violencia psicológica en la relación inmediata pasada o en la que actualmente mantenían. La violencia psicológica fue la que predominó en las alumnas de enfermería, psicología, turismo y derecho. El 34\% reportó que la violencia psicológica casi siempre estaba acompañada de otro tipo de violencia (física, económica o sexual). Sólo el $31 \%$ puedo reconocer aspectos de los tres tipos de violencia en su relación.

De acuerdo con Izquierdo (2007), los aspectos de la vida moderna afectan al desarrollo emocional de las personas y consecuentemente también a la armonía emocional de las parejas. Un estudio realizado por Blázquez, Moreno y García-Baamonde (2015) con 1080 estudiantes de la Universidad de Extremadura (España), con la finalidad de analizar la influencia que tiene el repertorio emocional individual (emotividad, eficacia, rigidez e ilusión) en la capacidad de infligir malos tratos psicológicos en la pareja, evidenció que los jóvenes con menores recursos emocionales suelen utilizar más la violencia psicológica basada en críticas, insultos, descalificaciones, minusvalorizaciones, hostilidad, desapego y manipulación, en sus relaciones de pareja. De igual forma, este estudio encontró correlaciones positivas entre los factores del maltrato psicológico con las competencias emocionales, a excepción de la competencia Nivel de rigidez, la cual no correlacionó con ningún factor de maltrato psicológico.

Un estudio llevado a cabo por Lazarevich, Irigoyen, Sokolava y Delgadillo (2013) con 729 estudiantes universitarios mexicanos, sobre la violencia en el noviazgo, la sintomatología depresiva y la autoestima, reportó que los participantes mostraron una elevada prevalencia de violencia verbalemocional. Las agresiones fueron tanto por parte de los hombres como de las mujeres. La conducta violenta se asoció con la baja autoestima en las mujeres, 
como síntomas depresivos en ambos sexos. La sugerencia dada por estos investigadores destaca la necesidad de desarrollar programas educativos orientados a la prevención de la violencia, el manejo de las emociones y la resolución de conflictos.

Rubio-Garay, Carrasco, Amor y López- González (2015) han evidenciado que el afecto negativo, basado en la ira y la hostilidad, facilita la agresión. De acuerdo con Rojas (2012) una de las características emocionales de las personas que tienen una participación activa en la violencia, muestran una incapacidad para regular la ira, la tristeza, la depresión y las frustraciones.

Marcus (como se citó en Blázquez-Alonso et al., 2012) por su parte, referencia, que las emociones positivas son un elemento inhibidor de la violencia en la pareja. Por lo que al parecer la gestión constructiva de la vida emocional puede llegar a determinar el ajuste personal y social del individuo. Extremera y Fernández-Berrocal (2003), enfatizan el papel de la inteligencia emocional como factor protector de la violencia al mostrar correlaciones negativas entre la conciencia y manejo de las propias emociones, con la justificación de la agresión y niveles elevados de impulsividad.

Así mismo, un estudio realizado por Pérez et al. (2018) destaca la necesidad de estudiar la asertividad vinculada a la inteligencia emocional como una instancia opuesta a la violencia, sugiriendo que ésta puede contribuir a la estabilidad en la pareja y el eventual matrimonio como parte de un procesos de felicidad centrado en la asertividad, la negociación y la corresponsabilidad.

De acuerdo con Gregorio y Corasi (2017) el noviazgo como fenómeno afectivo y social, sigue siendo asociado a una vivencia afectiva y emocional en la que se involucran sentimientos de confianza, felicidad, cariño, comprensión y valores de responsabilidad, sinceridad y compromiso, que van más allá de una concepción social o cultural asociado a la satisfacción sexual y económica. Dado lo anterior, el presente estudio tuvo como propósito identificar en qué porcentaje se presentaba la inteligencia emocional, el nivel de felicidad, enojo y violencia psicológica en el noviazgo en estudiantes de dos universidades; cómo la inteligencia emocional, el nivel de felicidad y el enojo se relacionan con la presencia de violencia psicológica en la relación de noviazgo de estudiantes universitarios. De igual forma se pretendió identificar los posibles contrastes entre dichas categorías de análisis a fin de hallar los posibles factores asociados que proporcionaran información pertinente para su oportuna atención en el ámbito educativo.

\section{Método \\ Participantes}

Participaron un total de 242 universitarios mexicanos, estudiantes de la licenciatura en psicología de dos universidades públicas del país (173 
mujeres y 63 hombres). Todos estudiantes de $1^{\circ}$ a $7^{\circ}$ semestre: el $86.4 \%$ de los participantes cursaba de $1^{\circ}$ a $3^{\circ}$ semestre y el $13.6 \%$ cursaba de $4^{\circ}$ a $7^{\circ}$ semestre. El rango de edad fue de 17 a 37 años, con una edad promedio de $M=19.3 ; D S=2.45$ años. 139 estudiantes eran de la universidad del Estado de Hidalgo (99 mujeres y 40 hombres) considerada para este estudio como una población urbana y 103 estudiantes de una universidad del Estado de Zacatecas, Campus Jalpa (78 mujeres y 25 hombres) considerada como una población semiurbana. Los participantes fueron seleccionados con base en un muestreo no probabilístico intencional.

\section{Diseño y tipo de estudio}

El estudio fue de tipo descriptivo con un diseño no experimental transaccional de alcance correlacional-comparativo.

\section{Variables e instrumentos}

Inteligencia emocional, fue medida con la Trait Meta-Mood Scale TMMS-24 validado para población mexicana por Rodríguez, Sánchez, Valdivia y Padilla en 2005 de la versión española de Extremera, FernándezBerrocal y Ramos (2004). Contiene tres dimensiones: a) atención a los propios sentimientos, b) claridad emocional y c) reparación de las propias emociones. Con una escala Likert dividida en cinco opciones que van desde "muy de acuerdo" a "muy desacuerdo". Tiene un Alfa de Cronbach de .090.

Felicidad, fue medida con el Cuestionario de Felicidad de Oxford (OHQ por sus siglas en inglés). Este cuestionario fue desarrollado por Hills y Argyle (2002) y mide de forma global la felicidad personal. Consta de 29 ítems con seis opciones de respuestas posibles en una escala Likert, las puntuaciones totales presentan un rango de 29 a 174 puntos, correspondiendo las puntuaciones más altas a mayores niveles de bienestar subjetivo. Tiene reactivos positivos (ejemplo "Me interesan muchísimo los demás") y negativos (ejemplo "No soy demasiado optimista con respecto al futuro"), siendo el mismo número de reactivos para ambos casos. Esta escala ha demostrado tener una alta consistencia interna. En su versión original presentó un Alfa de Cronbach de 0.90.

Enojo, fue evaluado con la Escala de Enojo Rasgo. Elaborado por Alcázar, Deffenbacher y Byrne (2011) para población mexicana, mide el grado en que una persona experimenta enojo. Consta de 10 ítems sobre la propensión o tendencia a molestarse a través del tiempo y las situaciones. Consta de dos subescalas: una subescala mide reacción del enojo ante situaciones frustrantes, la cual está conformada por 5 reactivos con un Alfa de Cronbach de .78-.79 y la otra mide el temperamento de enojo, tiene 5 reactivos con un Alfa de Cronbach de .79-.85.

Violencias en el noviazgo, fue medida con la Escala de violencia 
psicológica en el noviazgo, elaborada por Olmos y Dávalos-Romo (2016), consta de 75 ítems subdivididos en dos apartados distribuidos de la siguiente forma: En el primero, se encuentran todas las preguntas que requieren respuestas libres o con opciones múltiples (16 ítems): apartado de datos generales (5), Factores familiares: aspectos generales (4) y aspectos generales de la relación de noviazgo (7). El segundo grupo se divide en el apartado de Factores familiares (Relación Padre-Madre, Relación Madre, Relación Padre-Contigo, Relación Madre-Contigo), apartado de Violencia Psicológica en el noviazgo, Relación en el noviazgo (Relación Yo-Novia, Relación Novia (o)-Conmigo) y el apartado de Roles de género. La prueba tiene un Alfa de Cronbach de .89. Es importante aclarar que para esta investigación se tomaron en cuenta el apartado de Violencia psicológica en el noviazgo (12) y Relación en el noviazgo: Relación Yo-Novia/o (6), Relación Novia/o-Conmigo (7), dando un total de 25 ítems.

\section{Procedimiento}

Los participantes fueron informados del objetivo y contenido del estudio, así como de la confidencialidad de los resultados, y se solicitó su consentimiento informado previo a ser evaluados. Una vez que se contó con su autorización se aplicaron los instrumentos de medición de manera grupal, para ellos, cada universidad se encargó de asignar un responsable y llevarla a cabo. Al término de la aplicación se agradeció su colaboración.

\section{Análisis de datos}

Los datos fueron analizados en el Paquete Estadístico para las Ciencias Sociales (SPSS) versión 23. En un primer momento se realizó un análisis estadístico descriptivo con la finalidad de identificar en qué porcentaje se presentaba la inteligencia emocional, el nivel de felicidad, enojo y violencia en el noviazgo en estudiantes de las dos universidades participantes; posteriormente se llevó a cabo un análisis de muestras independientes $t$ de Student con el objetivo de identificar los posibles contrastes entre dichas variables, seguido de un análisis de correlación Rho de Spearman para identificar la relación entre estas variables.

\section{Resultados}

Resultados descriptivos de la medición por variables.

Inteligencia Emocional.

En cuanto a la medición de la inteligencia emocional obtenida con la Escala de Inteligencia Emocional TMMS-24, se encontró que en la dimensión de atención a los propios sentimientos, los estudiantes de la universidad de Hidalgo, obtiene el mayor porcentaje en la categoría de "adecuada percepción" (53.9\%); por su parte, los estudiantes de la universidad de 
Zacatecas, Campus Jalpa, presentan un mismo porcentaje en "presta poca atención" y "adecuada percepción” (45.6\%).

En la dimensión de comprensión emocional o claridad emocional, la mayoría de los estudiantes de la universidad de Hidalgo se encuentran en "debe mejorar su comprensión" (44.6\%) y la mayoría de los estudiantes de la universidad de Zacatecas, Campus Jalpa, se encuentran en una "adecuada comprensión" (47.5\%).

Finalmente, en la dimensión de regulación o reparación de las propias emociones, tanto los estudiantes de la universidad de Hidalgo como de la universidad de Zacatecas, Campus Jalpa, presentan una "adecuada regulación" con un $50.3 \%$ y $49.5 \%$ respectivamente (ver Tabla 1).

Tabla 1. Porcentaje de alumnos en el TMMS-24, por universidad y dimensión: Percepción emocional, comprensión emocional y regulación emocional

\begin{tabular}{clccc}
\hline Dimensión & & $\begin{array}{c}\text { Estudiantes } \\
\text { de Hidalgo }\end{array}$ & $\begin{array}{c}\text { Estudiantes de } \\
\text { Zacatecas }\end{array}$ \\
\hline $\begin{array}{c}\text { Atención a } \\
\text { los propios }\end{array}$ & $\begin{array}{l}\text { Debe mejorar su percepción: Presta poca } \\
\text { atención }\end{array}$ & $35.2 \%$ & $45.6 \%$ \\
& $\begin{array}{c}\text { Adecuada percepción } \\
\text { Debe mejorar su percepción: presta demasiada } \\
\text { atención }\end{array}$ & $53.9 \%$ & $45.6 \%$ \\
Claridad & Debe mejorar su comprensión & $40.7 \%$ & $0.8 \%$ \\
emocional & Adecuada comprensión & $39.5 \%$ & $45.6 \%$ \\
& Excelente comprensión & $15.8 \%$ & $47.5 \%$ \\
Reparación & Debe mejorar su regulación & $32.3 \%$ & $0.67 \%$ \\
de las propias & Adecuada regulación & $50.3 \%$ & $49.5 \%$ \\
emociones & Excelente regulación & $17.0 \%$ & $16.5 \%$ \\
\hline
\end{tabular}

Sin embargo, si se observan los puntajes promedio de cada una de estas dimensiones se puede apreciar que, los estudiantes de la universidad de Hidalgo, se encuentran dentro de un rango aceptable de atención emocional con una $\mathrm{M}=22.76$; $\mathrm{DE}=5.80$; y en el caso de los estudiantes de la universidad de Zacatecas, Campus Jalpa el puntaje promedio fue $\mathrm{M}=21$. 89, $\mathrm{DE}=6.45$, ubicándolos en un rango donde deben mejorar su atención, debido a que son poco capaces para sentir y expresar los sentimientos de forma adecuada (ver Tabla 2).

Siendo las mujeres las que suelen prestar una mayor atención a sus emociones que los hombres, al reportar 68 de ellas que están totalmente de acuerdo en comparación de 15 de ellos.

Respecto de los puntajes obtenidos en la dimensión de comprensión o claridad emocional los estudiantes de la universidad de Hidalgo obtuvieron una $\mathrm{M}=26.02 ; \mathrm{DE}=7.40$, lo cual representa que tienen una adecuada comprensión emocional. En cuanto a los estudiantes de la universidad de Zacatecas el puntaje se encontró con una $\mathrm{M}=25.84 ; \mathrm{DE}=6.80$ para el total de 
participantes, encontrándose éste dentro de los parámetros de un rango donde es preciso mejorar la comprensión emocional.

Las mujeres fueron quienes también presentaron una mayor frecuencia (30 de ellas) en estar totalmente de acuerdo en comprender sus sentimientos, en comparación con los hombres (9 de ellos). Viéndose también ellas más afectadas por sus sentimientos que los hombres.

Con relación a los puntajes obtenidos en la dimensión de regulación o reparación emocional, se encontró que los estudiantes de la universidad de Hidalgo tuvieron puntajes promedio de $\mathrm{M}=26.94$; $\mathrm{DE}=7.37$, lo cual representa que se encuentran dentro de una adecuada reparación. Los estudiantes de la universidad de Zacatecas por su parte, tuvieron una $\mathrm{M}=27.80 ; \mathrm{DE}=6.48$, dando como resultado que se encuentran dentro de los parámetros de una adecuada reparación de las emociones.

También se encontró que las mujeres (43 de ellas) son quienes más buscan cambiar su estado de ánimo, que los hombres. No obstante un número importante de hombres también busca hacerlo (25 de ellos).

Tabla 2. Media de puntajes obtenidos en el TMMS-24, por universidad y dimensión: Atención emocional, claridad emocional y reparación emocional

\begin{tabular}{llcc}
\hline Dimensión & Universidad & Media & $\begin{array}{c}\text { Desviación } \\
\text { estándar }\end{array}$ \\
\hline Puntaje Total de IE en área de Atención & Hidalgo & 22.76 & 5.80 \\
a los propios sentimientos & Zacatecas, Campus Jalpa & 21.89 & 6.45 \\
& Total & 25.62 & 6.99 \\
Puntaje Total de IE en área de Claridad & Hidalgo & 26.02 & 7.40 \\
emocional & Zacatecas, Campus Jalpa & 25.84 & 6.80 \\
& Total & 25.62 & 7.15 \\
Puntaje Total de IE en área de & Hidalgo & 26.94 & 7.37 \\
Reparación de las propias emociones & Zacatecas, Campus Jalpa & 27.80 & 6.48 \\
& Total & 27.31 & 7.01 \\
\hline
\end{tabular}

Al realizar un análisis comparativo de las medias por sexo con una prueba $t$ de Student, se encontró que no existen diferencias estadísticamente significativas entre hombres y mujeres, en las dimensiones medidas por el TMMS-24. Sin embargo con una análisis descriptivo (ver Tabla 3), se pudo identificar que en la dimensión Atención, las mujeres obtuvieron un valor promedio de $\mathrm{M}=22.81 ; \mathrm{DE}=5.98$, y los hombres un valor promedio de $\mathrm{M}=21.3$; DE=6.30, lo cual de acuerdo con los puntajes del TMMS-24, ambos, mujeres y hombres deben mejorar su atención, mostrando poca capacidad para sentir y expresar sus sentimientos de forma adecuada.

Así mismo, en cuanto a la dimensión Claridad, las mujeres tienen una $\mathrm{M}=25.70 ; \mathrm{DE}=7.18$, en comparación con los hombres quienes tuvieron una $\mathrm{M}=25.46 ; \mathrm{DE}=7.15$; esto significa que los hombres deben mejorar su claridad emocional a diferencia de las mujeres, pues al parecer presentan menor capacidad para comprender sus propios estados emocionales. 
En cuanto a la dimensión Reparación, las mujeres obtuvieron un puntaje promedio de $\mathrm{M}=27.33, \mathrm{DE}=7.18$, y los hombres una $\mathrm{M}=27.35$; $\mathrm{DE}=6.55$, lo cual de acuerdo con el TMMS-24, ambos sexos presentan una adecuada reparación siendo capaces de regular los estados emocionales correctamente.

Al llevar a cabo el análisis con la $t$ de Student, se encontró que no existen diferencias estadísticamente significativas en las medias obtenidas por los estudiantes de ambas universidades $t(240-232)=.222, p \leq .05$.

Tabla 3. Comparación por sexo del TMMS 24

\begin{tabular}{lcccc}
\hline Dimensión & Sexo & N & Media & $\begin{array}{c}\text { Desviación } \\
\text { estándar }\end{array}$ \\
\hline Atención a los propios & Femenino & 177 & 22.81 & 5.98 \\
sentimientos & Masculino & 64 & 21.31 & 6.30 \\
Claridad emocional & Femenino & 177 & 25.70 & 7.18 \\
& Masculino & 64 & 25.46 & 7.15 \\
Reparación de las propias & Femenino & 177 & 27.33 & 7.18 \\
emociones & Masculino & 64 & 27.35 & 6.55 \\
\hline
\end{tabular}

\section{Felicidad}

En cuanto a la emoción de felicidad medida con el Oxford Happiness Questionarie, se encontró que la mayoría de los alumnos de la universidad de Hidalgo se ubican en la categoría de "no particularmente felices o infelices" (86.3\%), mientras que la mayoría de los alumnos de la universidad de Zacatecas, Campus Jalpa, se sitúan en la categoría de "felices" (61.1\%), como se puede ver en Tabla 4.

De igual forma, se encontró que los estudiantes de ambas universidades caen en un rango de 4.5, ubicándose como bastante felices.

Tabla 4. Porcentaje de alumnos en el Oxford Happiness Questionarie, por universidad y nivel: algo feliz, no particularmente feliz o infeliz, feliz.

\begin{tabular}{lll}
\hline Nivel de felicidad & $\begin{array}{l}\text { Estudiantes } \\
\text { Hidalgo }\end{array}$ & $\begin{array}{l}\text { Estudiantes } \\
\text { Zacatecas }\end{array}$ \\
\hline Algo infeliz & $6,4 \%$ & $6.7 \%$ \\
No particularmente feliz o infeliz & $\mathbf{8 6 , 3 \%}$ & $32.0 \%$ \\
Feliz & $0,71 \%$ & $\mathbf{6 1 . 1 \%}$ \\
\hline
\end{tabular}

Al llevar a cabo la comparación de medias, no se encontraron diferencias estadísticamente significativas entre la universidad de Hidalgo (4.5) y la universidad de Zacatecas, Campus Jalpa (4.5), según los resultados obtenidos a través de la $t$ de Student, $\quad t(240-181.19)=-1.89, p \leq .05$.

Escala de Enojo Rasgo.

Los resultados en cuanto a la escala de enojo, señalan que el mayor porcentaje de alumnos, tanto de la universidad de Hidalgo (43.8\%) como de la universidad de Zacatecas, Campus Jalpa (36.8\%), se encuentran en el nivel 
bajo (ver Tabla 5).

Tabla 5. Porcentaje de alumnos en la Escala de Enojo Rasgo, por universidad y nivel: bajo, promedio, alto

\begin{tabular}{lll}
\hline Nivel de enojo & $\begin{array}{l}\text { Estudiantes } \\
\text { Hidalgo }\end{array}$ & $\begin{array}{l}\text { Estudiantes } \\
\text { Zacatecas }\end{array}$ \\
\hline Bajo & $43,8 \%$ & $36.8 \%$ \\
Promedio & $41,0 \%$ & $30.0 \%$ \\
Alto & $15,1 \%$ & $31.0 \%$ \\
\hline
\end{tabular}

En cuanto a la comparación entre los estudiantes de ambas universidades mediante un análisis estadístico de $t$ Student, se encontró que no existen diferencias estadísticamente significativas entre los estudiantes de la universidad de Hidalgo y los estudiantes de la universidad de Zacatecas (media de puntajes universidad Hidalgo 18.05; universidad de Zacatecas, Campus Jalpa 19.49), $t(240-181.19)=-1.89, p \leq .05$.

Escala de violencia psicológica en el noviazgo.

Se realizó un análisis de frecuencias para identificar cuál era el comportamiento de las respuestas en cuanto a los indicadores de violencia en las relaciones de noviazgo, de lo cual se observó, que éstas muestran un mayor porcentaje en la categoría de "nunca", como ausencia de violencia; no obstante, es importante destacar los porcentajes que refieren la presencia de la misma con menor frecuencia, en las categorías de "casi nunca", "casi siempre" y "siempre" (ver Tabla 6).

Tabla 6. Porcentaje de alumnos en la escala de violencia en el noviazgo. Datos por universidad y grado de frecuencia: nunca, casi nunca, casi siempre y siempre.

\begin{tabular}{llllll}
\hline Relaciones familiares & Universidades & Nunca & $\begin{array}{l}\text { Casi } \\
\text { nunca }\end{array}$ & $\begin{array}{l}\text { Casi } \\
\text { siempre }\end{array}$ & Siempre \\
\hline 1. Cuando YO me molesto con mi & Hidalgo & $74.2 \%$ & $18.8 \%$ & $5.5 \%$ & $1.6 \%$ \\
novio/a: le grito & Zacatecas & $63.0 \%$ & $28.3 \%$ & $8.7 \%$ & $0.0 \%$ \\
2. Cuando YO me molesto con mi & Hidalgo & $96.1 \%$ & $3.9 \%$ & $0.0 \%$ & $0.0 \%$ \\
novio/a: le prohíbo salir & Zacatecas & $82.6 \%$ & $15.2 \%$ & $1.1 \%$ & $1.1 \%$ \\
3. Cuando YO me molesto con mi & Hidalgo & $91.4 \%$ & $7.0 \%$ & $0.8 \%$ & $0.8 \%$ \\
novio/a: lo/la jaloneo o empujo & Zacatecas & $90.2 \%$ & $7.6 \%$ & $2.2 \%$ & $0.0 \%$ \\
4. Cuando YO me molesto con mi & Hidalgo & $42.3 \%$ & $39.2 \%$ & $11.5 \%$ & $6.9 \%$ \\
novio/a: me marcho & Zacatecas & $47.3 \%$ & $24.7 \%$ & $22.6 \%$ & $5.4 \%$ \\
5. Cuando YO me molesto con mi & Hidalgo & $93.0 \%$ & $4.7 \%$ & $1.6 \%$ & $0.8 \%$ \\
novio/a: lo/la insulto & Zacatecas & $85.9 \%$ & $9.8 \%$ & $4.3 \%$ & $0.0 \%$ \\
6. Cuando YO me molesto con mi & Hidalgo & $94.5 \%$ & $5.5 \%$ & $0.0 \%$ & $0.0 \%$ \\
novio/a: tiro o golpeo objetos & Zacatecas & $94.6 \%$ & $4.3 \%$ & $1.1 \%$ & $0.0 \%$ \\
7. Cuando MI NOVIO/A se & Hidalgo & $76.7 \%$ & $20.2 \%$ & $31 \%$ & $0.0 \%$ \\
molesta conmigo: me grita & Zacatecas & $69.9 \%$ & $25.8 \%$ & $4.3 \%$ & $0.0 \%$ \\
8. Cuando MI NOVIO/A se & Hidalgo & $91.4 \%$ & $5.5 \%$ & $1.6 \%$ & $1.6 \%$ \\
molesta conmigo: me prohíbe & Zacatecas & $83.7 \%$ & $13.0 \%$ & $2.2 \%$ & $1.1 \%$ \\
salir & & & & \\
9uando MI NOVIO/A se & Hidalgo & $92.2 \%$ & $6.2 \%$ & $1.6 \%$ & $0.0 \%$ \\
molesta conmigo: me jalonea o & Zacatecas & $91.3 \%$ & $5.4 \%$ & $3.3 \%$ & $0.0 \%$
\end{tabular}
empuja 
10. Cuando MI NOVIO/A se molesta conmigo: llora

11. Cuando MI NOVIO/A se molesta conmigo: se marcha

12. Cuando MI NOVIO/A se molesta conmigo: me insulta

13. Cuando MI NOVIO/A se molesta conmigo: tira o golpea objetos

$\begin{array}{llll}69.8 \% & 17.8 \% & 9.3 \% & 3.1 \% \\ 67.4 \% & 26.1 \% & 5.4 \% & 1.1 \% \\ 57.7 \% & 25.4 \% & 14.6 \% & 2.3 \% \\ 56.5 \% & 25.0 \% & 12.0 \% & 6.5 \% \\ 93.8 \% & 6.3 \% & 0.0 \% & 0.0 \% \\ 89.0 \% & 6.6 \% & 3.3 \% & 1.1 \% \\ 92.2 \% & 7.8 \% & 0.0 \% & 0.0 \% \\ 93.4 \% & 4.4 \% & 1.1 \% & 1.1 \%\end{array}$

Siguiendo la idea anterior, y considerando, como se señalo previamente, que si un joven hace mención de que el indicador de violencia se presenta en la categoría de "casi nunca", "casi siempre" o "siempre", es indicio de que en sus relaciones de noviazgo sí se presenta algún tipo de violencia (con mayor o menor frecuencia, pero sí la hay), en la Tabla 7, se muestra la suma de los porcentajes de estas tres categorías comparadas con la categoría de "nunca". Con esto, concentramos dos únicas categorías: "No violencia" y "Si violencia".

Como primer punto importante, se observa que la mayoría de los indicadores obtienen un porcentaje mayor en "No violencia"; sin embargo, al analizar el apartado de "Si violencia", el porcentaje menor es de 3.9\%; considerando con eso, que a mayor o menor grado, se presenta algún tipo de violencia en todos los indicadores explorados.

Al analizar las universidades, el indicador de "cuando yo me molesto con mi novio/a: me marcho" es el único que presenta un porcentaje mayor en "Si violencia" (57.6\% en la universidad de Hidalgo y $52.7 \%$ en la universidad de Zacatecas). Al respecto es importantes considerar que a pesar del contexto en el que se encuentran los alumnos (urbano y semiurbano), el indicador que prevalece es el mismo.

Tabla 7. Porcentaje de alumnos en la escala de violencia en el noviazgo. Datos por universidad y grado de frecuencia: no violencia, si violencia.

\begin{tabular}{lllll}
\hline Relaciones familiares & Universidades & $\begin{array}{l}\text { No } \\
\text { violencia }\end{array}$ & $\begin{array}{l}\text { Si } \\
\text { Violencia }\end{array}$ \\
\hline 1. Cuando YO me molesto con mi & $\begin{array}{l}\text { Hidalgo } \\
\text { novio/a: me marcho }\end{array}$ & $\mathbf{4 2 . 3 \%}$ & $\mathbf{5 7 . 6 \%}$ \\
& Zacatecas & $\mathbf{4 7 . 3 \%}$ & $\mathbf{5 2 . 7 \%}$ \\
\hline
\end{tabular}

Relación de inteligencia emocional, felicidad y enojo con violencia psicológica en el noviazgo.

En este apartado se exponen las relaciones estadísticas que dan evidencia de los vínculos de los estudiantes universitarios.

Las correlaciones, se llevaron a cabo con la estrategia de Rho de Spearman a una confiabilidad del $99 \%$, para una población de universitarios de 242 alumnos. 
De acuerdo con la Tabla 8 se observa que la felicidad está vinculada en forma directa con la regulación de las emociones y la inteligencia emocional de los estudiantes, así como al incrementarse la felicidad en estos alumnos, se muestran más conformes con algunas creencias sobre las relaciones de pareja.

Es muy probable que los estudiantes que se describen a sí mismos como felices presenten una mejor inteligencia emocional, particularmente la referida a su propio conocimiento y capacidad para reparar en las propias emociones.

Por el contrario, se puede apreciar que a medida que existe una relación inversamente proporcional entre el enojo y los componentes de la inteligencia emocional, siendo así que a medida que el enojo aumenta, existe una menor comprensión emocional, así como una menor regulación emocional por parte de los participantes, de igual forma, a medida que aumenta la relación de violencia del participante como Yo Novia(o), el enojo también se incrementa y disminuye la felicidad. Así mismo, se puede observar que a medida que aumenta la violencia psicológica en el noviazgo, la felicidad se ve disminuida en la muestra de estudiantes participantes.

Tabla 8. Relación de las emociones de los estudiantes universitarios con categorías del TMMS y el cuestionario de violencia en el noviazgo

\begin{tabular}{lll}
\hline Variables & Felicidad & Enojo \\
Atención & $0.138^{*}$ & \\
Comprensión-Claridad emocional & $0.426^{* *}$ & $-0.195^{* *}$ \\
Regulación-Reparación de las propias emociones & $0.477^{* *}$ & $-0.210^{* *}$ \\
Violencia psicológica en el noviazgo & $-0.209^{* *}$ & \\
Relación de violencia Yo Novia(o) & $-0.134^{*}$ & $0.130^{*}$ \\
\hline
\end{tabular}

$$
\text { Nota: Rho de Spearman, alfa } .01^{* *}, .05^{*}, \mathrm{n}=242
$$

De acuerdo con lo expuesto en la Tabla 9, se puede observar que, al incrementarse la edad de los alumnos universitarios, disminuye la expresión de la felicidad y aumenta la expresión de violencia psicológica. Esto, probablemente dada la carga de responsabilidades que tengan que hacer en lo cotidiano de sus vidas y de sus relaciones interpersonales.

Tabla 9. Relaciones con la edad de los estudiantes universitarios

\begin{tabular}{lc}
\hline Variables & Edad \\
\hline Felicidad & $-0,19$ \\
Violencia psicológica & 0,17 \\
\hline Nota: Rho Spearman, alfa $.01, \mathrm{n}=242$
\end{tabular}

\section{Discusión}

Este estudio abordó el fenómeno de la violencia psicológica en el noviazgo, asociado a la emoción del enojo, la inteligencia emocional y el bienestar psicológico, por lo que se plantearon tres objetivos: a) identificar en 
qué porcentaje se presentaba la inteligencia emocional, el nivel de felicidad, enojo y violencia psicológica en el noviazgo en estudiantes de las dos universidades; b) identificar cómo la inteligencia emocional, el nivel de felicidad y el enojo se relacionan con la presencia de violencia en la relación de noviazgo de estudiantes universitarios y, c) identificar los posibles contrastes entre dichas variables.

Los resultados permiten identificar que el mayor porcentaje de los estudiantes de la universidad de Hidalgo y la universidad de Zacatecas, Campus Jalpa, presentan una adecuada conciencia sobre sus propias emociones, buena capacidad para reconocer sus sentimientos y lo que éstos significan, así como una adecuada regulación de las mismas. De acuerdo con Taramuel y Zapata (2017), cuando se presentan puntajes aceptables en estas dimensiones las personas son capaces de distinguir con más claridad sus emociones, de entender cómo evolucionan y cómo se integran a sus pensamientos, además de prestar mayor atención a sus emociones y a sus sentimientos.

Así mismo refieren Fernández-Berrocal y Extremera (2009), que cuando las personas muestran la habilidad para percibir, expresar y regular de forma adecuada sus emociones éstas pueden llegar a presentan un estado de bienestar subjetivo. Quizá por eso los participantes de este estudio, principalmente los estudiantes de Zacatecas, reportaron sentirse felices y aunque los estudiantes de la universidad de Hidalgo, manifestaron no sentirse particularmente felices o infelices, tampoco reportan un estado de infelicidad.

Es importante destacar como refieren Blázquez-Alonso et al. (2012), que la inteligencia emocional suele correlacionar positivamente con el autocontrol, la empatía, el bienestar subjetivo en situaciones sociales como en las relaciones afectivas con la pareja, hecho que también se puede observar en el caso de algunos estudiantes en el presente estudio.

Por otra parte, es importante señalar que probablemente algunos de los estudiantes de la universidad de Zacatecas, Campus Jalpa como de Hidalgo, tanto hombres como mujeres deben mejorar y trabajar sobre sus propias emociones, particularmente la comprensión emocional, la cual presentó un porcentaje ligeramente más elevado en esta dimensión. Encontrando también diferencias entre hombres y mujeres, siendo los hombres quienes deben trabajar la comprensión emocional. Por lo que se propone mejorar esta habilidad en este grupo de estudiantes debido a que se encuentran estudiando la licenciatura en psicología y es importante para poder orientar a otros. Además resulta indispensable el poder comprender qué pasa con los propios sentimientos y los de los demás, así como poder indagar los motivos por los cuales presentan un puntaje parcialmente más alto en esta dimensión que en las otras.

De igual forma, no se encontraron diferencias estadísticamente 
significativas entre los estudiantes de ambas universidades en cuanto a dichas habilidades emocionales, como tampoco entre las emociones de felicidad, enojo y violencia psicológica. Parece ser que al menos en este estudio, el pertenecer a un contexto urbana o semiurbana no marca una diferencia relevante en la manifestación, percepción, expresión y regulación de las emociones como en la presencia de violencia psicológica en el noviazgo; sin embargo, es de observar que existe la presencia de un nivel de enojo alto, particularmente en los estudiantes de la universidad de Zacatecas, Campus Jalpa, el cual quizá podría considerarse como detonante de violencia en el caso de los estudiantes que se encuentran dentro del porcentaje de violencia, en la relación por parte del participante hacia el novio(a) como del novio(a) hacia el participante, tal como se puede apreciar en los datos descriptivos y en el análisis de correlación.

Es de llamar la atención la conducta que suelen presentar los estudiantes de ambas universidades cuando se enojan o discuten con su pareja, quienes suelen retirarse del lugar. Esto podría tener dos interpretaciones, una que se encuentra asociada a la regulación emocional, y otra que podría estar vinculada a la reacción de violencia psicológica que suele también, ser otra forma de manifestación, por lo que se sugiere seguir investigando sobre esta asociación.

Así también, el hecho de que los estudiantes tanto de la universidad de Hidalgo como de Zacatecas, se encuentren en un nivel de enojo promedio y un nivel de felicidad alto, puede ser un factor importante para que aparentemente no se reportara una mayor frecuencia de violencia. De hecho como refiere Marcus y Swett (2002, como se citó en Blázquez, Moreno, \& García-Baamonde, 2015) las emociones positivas son un elemento inhibidor de la violencia en la pareja. Así también, Extremera y Fernández-Berrocal (2002), enfatizan el papel de la inteligencia emocional como factor protector de la violencia al mostrar correlaciones negativas entre la conciencia y manejo de las propias emociones, con la justificación de la agresión y niveles elevados de impulsividad.

En cuanto a la violencia psicológica en el noviazgo, es importante señalar que aunque los porcentajes son bajos, si se presenta de una u otra forma con frecuencia algún tipo de violencia, por ello, tal y como lo refiere González, Echeburua y Corral (2008, como se citó en Betancourt, López, \& González, 2011), no puede dejarse de lado, dado que es muy importante tomar acciones promoviendo la regulación emocional del enojo, fortaleciendo el bienestar psicológico y de esta forma prevenir la violencia en el noviazgo.

\section{Conclusión}

Este estudio contribuye en el campo de la psicología a entender que las emociones y la violencia en el noviazgo están estrechamente relacionadas, por 
lo que es conveniente desarrollar acciones preventivas entorno a la regulación emocional que contribuyan en la prevención de la misma.

Así mismo, tener presente que las habilidades emocionales pueden ayudar a fortalecer interacciones positivas y sanas en las relaciones de noviazgo, siendo el enojo una de las emociones a las que habrá de prestar atención en futuras investigaciones.

Se considera que una de las limitaciones de este estudio, es que el instrumento de violencia psicológica, no permite identificar los motivos de porqué se retiran el participante o el novio(a) cuando se molestan, por ejemplo. No obstante este estudio, aproxima a comprender en qué se necesita prestar atención para prevenir la violencia en el noviazgo.

Finalmente, es importante resaltar el compromiso que poseen las universidades, detectando y brindando mayores recursos emocionales a los futuros psicólogos, llevándolos a ser más creativos, felices y con relaciones personales y de pareja sanas, detectando cualquier señal de violencia ejecutada o recibida por su entorno, sin que esta llegue a ser normalizada.

\section{References:}

1. Alberdi, I. (2005). Cómo reconocer y cómo erradicar la violencia contra las mujeres. En Obra Social "la Caixa". Violencia: Tolerancia Cero. Barcelona: Fundación La Caixa.

2. Alcázar, R. J., Deffenbacher, J. L., \& Byrne, Z. S. (2011). Assessing the factor structure of the anger expression inventory (ML-STAXI) in a Mexican sample. International Journal of Psychology and Psychological Therapy, 11, 307-318.

3. Batiza, F.J. (2017). La violencia de pareja. Un enemigo silencioso. Archivos de Criminología, seguridad privada y criminalística, 4(8), 144-151. Recuperado de https://dialnet.unirioja.es/servlet/articulo?codigo=5813533

4. Betancourt, D., López, M., \& González, D. M. (2011). Violencia en el noviazgo.

https://www.anahuac.mx/mexico/files/investigacion/2011/novdic/54.pdf

5. Blázquez-Alonso, M., Moreno-Manso, J.M., García-Baamonde. M. E., \& Guerrero-Barona, E. (2012). La competencia emocional como recurso inhibidor para la perpetración del maltrato psicológico en la pareja. Salud Mental, 35(4), 287-296.

6. Blázquez, M., Moreno, J.M., \& García-Baamonde, M.E. (marzo, 2015). Maltrato psicológico en las relaciones de pareja. La inteligencia emocional como factor protector y diferencias de género. Boletín de psicología, 113, 29-47. 
7. Cornelius, T. L., \& Resseguie, N. (2007). Primary and secondary prevention programs for dating violence: A review of the literature. Aggression and Violent Behavior, 12, 364-375.

8. Extremera, N., \& Fernández-Berrocal, P. (2003). La inteligencia emociona en el contexto educativo: hallazgos científicos de sus efectos en el aula. Revista de educación, 332, 97-116.

9. Fernández-Berrocal, P., \& Extremera, N. (2009). La inteligencia emocional y el estudio de la felicidad. Revista Interuniversitaria de formación del profesorado, 66, 85-108.

10. Extremera, N., Fernández-Berrocal, P., \& Ramos, N. (2004). Desarrolla tu inteligencia emocional. Barcelona, España: Kairós.

11. Guerri, M. (2016). Inteligencia emocional: una guía para mejorar tu vida. México: Meta Ediciones.

12. Gregorio, M. M., \& Corasi, A. C. (2017). El noviazgo en la Universidad. Recuperado

https://www.revistadifusiones.net/index.php/difusiones/article/view/8 $3 / 165$

13. Hills P., \& Argyle, M. (2002). The Oxford Happiness Questionnaire: a compact scale for the measurement of psychological well-being. Personality and Individual Differences, 33, 1073-1082.

14. Izquierdo, C. (2007). Crecer en el noviazgo. Lima, Perú: Paulinas.

15. Lazarevich, I., Irigoyen, M.E., Sokolava, A, V., \& Delgadillo, H. (2013). Violencia en el noviazgo y salud mental en estudiantes universitarios mexicanos. Recuperado de https://doi.org/10.1177/1757975913499032

16. Mesta, M. (2016). 9 de cada 10 adolescentes sufren violencia en noviazgo: OMS. Milenio. Recuperado de https://www.milenio.com/policia/9-10-adolescentes-sufren-violencianoviazgo-oms

17. Olmos, M., \& Dávalos-Romo, M.T. (2016). Desarrollo y validación de un cuestionario de violencia psicológica en el noviazgo (Tesis de pregrado). Universidad Autónoma de Zacatecas, México.

18. Olvera, J.A., Arias, J., \& Amador, R. (2012). Tipos de violencia en el noviazgo de estudiantes universitarias de la UAEM, Zumpango. Revista Electrónica de Psicología Iztacala, 15(1), 150-171.

19. Pérez, G., García, L., \& Pérez, M. I. (2018). Felicidad en el noviazgo en las relaciones de pareja libres de violencia y asertivas. Alternativas en psicología, 40, 128-140.

20. Pick, S., \& Givaudan, M. (2010). Prevención y detección temprana de violencia en el noviazgo; el caso de México. Universidad Nacional Autónoma de México, IMIFAP. Recuperado de 
http://toolsescuelas.com.mx/wpcontent/uploads/2014/05/11_Violencia-en-el-noviazgo-Brasilia.pdf

21. Pozueco, J.M., Moreno, J.M., Blázquez, M., \& García-Baamonde, M. E. (2013). Psicópatas integrados/subclínicos en las relaciones de la pareja: perfil, maltrato psicológico y factores de riesgo. Papeles del Psicólogo, 34(19), 32-48.

22. Ramírez, C.A., \& Núñez, D. A. (2010). Violencia en la relación de noviazgo en jóvenes universitarios: un estudio exploratorio. Enseñanza e investigación en psicología, 15 (2), 273-283.

23. Rey, C. A (2008). Prevalencia, factores de riesgo y problemáticas asociadas con la violencia en el noviazgo: Una revisión de la literatura. Avances en Psicología Latinoamericana, 26 (2), 227-241.

24. Rojas, B. (2012). Violencia en el noviazgo. Estrategias para el cumplimiento de la misión, 9(2), 102-116. Recuperado de https://revistas.upeu.edu.pe/index.php/r_estrategias/article/view/359/ 350

25. Rodríguez, M. A.; Sánchez, M. P.; Valdivia, J. A. \& Padilla, V. M. (abril, 2005). Perfil de inteligencia emocional en estudiantes universitarios de la Facultad de psicología. Trabajo presentado en el XXXII Congreso y LXXVI Asamblea del Consejo Nacional para la Enseñanza e Investigación en Psicología, Mexicali, México. Resumen recuperado

https://www.google.com/search?q=rodriguez+Nieto,+M.+A.;+S\%C3 $\%$ A1nchez+Miranda,+M.+P.;+Valdivia+V\%C3\%A1zquez,+J.+A.+y + Padilla+Montemayor,+V.+M.+(2005).+Perfil+de+inteligencia+emo cional+en+estudiantes+universitarios+de+la+Facultad+de+psicolog $\%$ C3\% ADa\&biw $=1920 \&$ bih $=937 \&$ tbm $=$ isch\&source $=$ iu\&ict $=1 \&$ fir $=\mathrm{Qqv027v2CKIEJM} \% 253 \mathrm{~A} \% 252 \mathrm{Ct6QeMJ1MAk031M} \% 252 \mathrm{C} \_\& v e t$ $=1 \&$ usg=AI4_-kTgnJ-

kVZjf3DB6LCgG716PICzbFQ\&sa=X\&ved=2ahUKEwiProulxJL1Ah UFJzQIHbr1DjQQ9QEwBnoECAkQBg\#imgrc=Qqv027v2CKIEJM:

26. Rubio-Garay, F. Carrasco, M.A., Amor, P., \& López- González, M.A. (2015). Factores asociados a la violencia en el noviazgo entre adolescentes: una visión crítica. Anuario de Psicología Jurídica, 25, 47-56.

27. Taramuel, J.A., \& Zapata, V.H (2017). Aplicación del test TMMS-24 para el análisis y descripción de la Inteligencia Emocional considerando la influencia del sexo. Revista Publicando, 11(1), 162181.

28. Valencia-Valera, A., \& Vega-López, M.G. (2015). Violencia en el noviazgo en adolescentes. Un problema de salud pública. Revista Salud Jalisco, 3, 164-168. 